\title{
Long Term Speech Perception Outcomes of Cochlear Implantation in Gap Junction Protein Beta 2 Related Hearing Loss
}

\author{
Sung Hee Kim ${ }^{1}$, Rajendra Nepali ${ }^{2}$, Myung Hoon Yoo ${ }^{3}$, Kwang-Sun Lee ${ }^{1}$, and Jong Woo Chung ${ }^{1}$ \\ ${ }^{1}$ Department of Otolaryngology, Asan Medical Center, University of Ulsan College of Medicine, Seoul, Korea \\ ${ }^{2}$ Department of Otolaryngology-Head and Neck Surgery, Gandaki Medical College, Pokhara, Nepal \\ ${ }^{3}$ Department of Otolaryngology-Head and Neck Surgery, Korea University College of Medicine, Seoul, Korea
}

\section{Received February 27, 2017 \\ Revised May 22, 2017 \\ Accepted May 24, 2017}

\section{Address for correspondence Jong Woo Chung, MD, PhD \\ Department of Otolaryngology, Asan Medical Center, \\ University of Ulsan \\ College of Medicine, \\ 88 Olympic-ro 43-gil, \\ Songpa-gu, Seoul 05505, Korea \\ Tel +82-2-3010-3718 \\ Fax +82-2-489-2773 \\ E-mail gfinder.jw@gmail.com}

Background and Objectives: The mutation of the gap junction protein beta 2 (GJB2) gene is the predominant cause of autosomal recessive non-syndromic hearing loss. The purpose of this study was to evaluate the speech perception outcome after cochlear implantation according to the presence of a GJB2 mutation. Subjects and Methods: During the period from March 2004 to February 2005, 38 patients underwent cochlear implantation at Asan Medical Center. Genetic factors and speech perception were evaluated in all subjects, and the patients were grouped according to the presence of a GJB2 mutation. The two groups were carefully matched according to the age at cochlear implantation. We analyzed four mutations in the GJB2 gene: 35delG, 167delT, 235delC, and E114G. Speech perception outcomes were measured using the open set, 1 and 2 syllables, the comprehension test, the Meaningful Auditory Integration Scale, the categories of auditory performance, and the Speech Intelligibility Rating scores. The evaluations were performed before the operation, 6 and 12 months thereafter, and then annually up to nine years after cochlear implantation. Results: Fifteen patients had bi-allelic GJB2 mutations (11 with $\mathrm{E} 114 \mathrm{G}$ and 4 with $235 \mathrm{delC}$ ), whereas the remaining 23 had wild type alleles. For the age-matched analysis, 14 patients were selected and divided into two groups of 7 subjects each: GJB2 mutation and no mutation (i.e., deafness of unknown origin). Overall, all patients showed improvement of speech perception outcome after cochlear implantation. There was no difference in the improvement between patients with and without GJB2 mutations at the 5-year and 9-year follow up. The pattern of improvement throughout the duration of the follow-up also showed no difference between the two groups. Conclusions: Similar outcomes of speech perception are expected after cochlear implantation in pediatric patients with or without GJB2 mutation.

J Audiol Otol 2017;21(2):95-102

KEY WORDS: Hearing loss · Cochlear implantation · Speech perception.

\section{Introduction}

Sensorineural hearing loss (SNHL) is a common congenital defect, and profound bilateral SNHL affects 1 in 1,000 children $[1,2]$. Cochlear implantation (CI) is an effective solution enabling patients with profound SNHL to achieve auditory-

This is an Open Access article distributed under the terms of the Creative Commons Attribution Non-Commercial License (http://creativecommons.org/licenses/by-nc/4.0/) which permits unrestricted non-commercial use, distribution, and reproduction in any medium, provided the original work is properly cited. verbal communication, regardless of the etiology (genetic or acquired). In a recently published meta-analysis, factors influencing $\mathrm{CI}$ outcomes were reported to be age at implantation, gap junction protein beta 2 (GJB2) mutations, inner ear malformations, and meningitis [3]. However, there remains a need to determine other prognostic factors because the influence of the above factors cannot account for all of the variance in speech performance following CI.

The GJB2 gene, encoding the protein connexin 26 (Cx26), is frequently found to be mutated in SNHL [4]. Among patients 
with autosomal recessive nonsyndromic hearing loss, GJB2 mutations have been found to be a major etiological factor in nonsyndromic deafness [5-7]. In Korea, 13.6\% patients with congenital hearing loss have a GJB2 mutation [8].

GJB2 is located on chromosome $13 \mathrm{q} 11$, and its autosomal recessive locus is designated as $D F N B 1$. As a result of the mutations at the DFNB1 locus, patients show prelingual hearing loss, especially in the high frequencies, with variable severity from mild to profound, but not progressive $[9,10]$. GJB2-related hearing loss is regarded as a result of altered potassium recirculation in the cochlea. Accumulation of potassium in the endolymph causes sensory hair cell dysfunction without involvement of the auditory nerve $[11,12]$. Based on these pathophysiological alterations in patients harboring a specific type of GJB2 mutation, DFNB1 (autosomal recessive) but not DFNA3 (autosomal dominant), better outcomes would be expected with $\mathrm{CI}$ than in patients affected by deafness because of other etiologies.

It is clear that disorders causing damage to the neural system, such as meningitis or auditory nerve pathology, may result in poorer CI outcomes when compared to the sensory hair cell damage observed in patients with a GJB2 mutation. Kang, et al. [13], studied CIs in young children with cochlear nerve deficiency diagnosed by magnetic resonance imaging (MRI). Speech Intelligibility Rating (SIR) scores in the control group gradually increased after CI, but the score in the study group were not changed even after CI. In open-set one-syllable tests, five out of six patients with cochlear nerve deficiency showed no response at the latest follow-up; therefore, the authors concluded that young children with nonvisualized auditory nerve on MRI showed worse outcomes than children with normal cochlear nerves.

However, reports on the post-CI speech outcomes in patients with GJB2 mutations are controversial. Davcheva-Chakar, et al. [14], reported results from 2 years' follow-up after $\mathrm{CI}$ in children with GJB2-related deafness and a control group of children with deafness of unknown etiology. Both groups showed similar improvements in speech perception scores after CI. Statistical analysis did not confirm significant differences between the groups 12 and 24 months after CI. On the other hand, Popov, et al. [15], reported that children with GJB2 mutations showed significantly better outcomes in a speech perception test than age-matched children with non-GJB2-related deafness over 3 years' follow-up after CI. In this retrospective study, we evaluated the speech perception outcomes following CI in pediatric patients with prelingual GJB2-related deafness over a long-term follow-up.

\section{Subjects and Methods}

\section{Subjects and selection criteria}

In the period from March 2004 to February 2005, CI was performed on 38 prelingually deafened children under 15 years of age at Asan Medical Center, Seoul, Korea. Blood was collected from all patients for genetic testing after obtaining signed informed consent from the parents and patients. All the children were ethnically Korean, and their native language was Korean.

For the analysis of speech outcomes, 10 children who had an inner ear anomaly and/or developmental difficulties were excluded. Inner ear anomalies were evaluated by high-resolution computed tomography and MRI. Overall, 28 patients were finally included in our study cohort. Two study protocols were approved by the Institutional Review Board (IRB No. 2014-0997, 2004-0121) of Asan Medical Center. One was a prospective protocol for collecting blood for genetic analysis and clinical data at 1 year after CI. The informed consent form was reviewed and approved by the IRB. The other study protocol involved retrospective review of the clinical data at 8 year later from the prospective study. The requirement for informed consent from each patient was waived for this analysis.

\section{Genetic analysis}

Genomic DNA was isolated from peripheral blood using the QIAamp DNA Blood Kit (Qiagen, Hilden, Germany). The coding region of the gene was amplified in two separate PCR assays and subsequently sequenced using the BigDye Terminator v3.1 Cycle Sequencing Ready Reaction Kit (Applied Biosystems, Foster City, CA, USA) and electrophoresis on ABI 3130 apparatus (Applied Biosystems).

\section{Auditory and speech evaluations}

All patients received audiometric examinations such as play audiometry, pure tone audiometry, auditory brainstem response, and otoacoustic emissions. In addition, the patients underwent a battery of cognitive, neurological, and psychological [Social Maturity Scale (SMS)] tests. Speech perception outcomes were assessed using open-set one- and two-syllable tests [16], a comprehension test [16], the Meaningful Auditory Integration Scale (MAIS) [17], SIR [18], and the Categories of Auditory Performance (CAP) [19]. The open-set tests included a one-syllable, consonant-vowel-consonant isophonemic meaningful word lists and two-syllable isophonemic meaningful word lists. The comprehension test assesses the recognition of a proposed sentence by a covered mouth. All measurements were scored before the operation, at 6 and 12 months thereafter, and then annually up to 9 years after CI. 


\section{Statistical analysis}

In the pediatric population, age is a factor in speech perception outcomes after CI. We therefore analyzed the relevant data in two ways. One was by matching the age at implantation, and the other was by analyzing the data from the patients under the age of 5 years at the time of CI. To estimate the effects of both time (duration of implant use) and group (GJB2 mutation and no mutation) on the speech outcomes of CI (openset one- and two-syllable, comprehension, MAIS, SIR, and CAP scores), we used a linear mixed model that accounted for patient effects. If the group-by-time interaction effect was significant, the outcomes were compared among the group effects within time points. If the group-by-time interaction effect was not significant, we excluded the group-by-time interaction effect from the analysis and compared the overall group effects for the outcomes. All reported $p$ values were two sided, and $p<0.05$ was considered to indicate statistical significance. The Mann-Whitney test was used to compare the results of the last follow-up (at 6-9 years of age). To accommodate the ceiling effects of the speech perception results, data were transformed into rationalized arcsine unit (RAU) scores [20]. Data manipulation and statistical analyses were conducted using SAS ${ }^{\circledR}$ version 9.3 (SAS Institute Inc., Cary, NC, USA), and all statistical analyses were performed in cooperation with a professional statistician.

\section{Results}

\section{GJB2 mutations and patient characteristics}

Among the $38 \mathrm{CI}$ patients initially screened in this study, 15 had biallelic GJB2 mutations. The remaining 23 had wild-type alleles. Table 1 lists the clinical features of the two groups. The male-to-female ratio was $12(80 \%)$ to $3(20 \%)$ in the GJB2 mutation group and $14(60.8 \%)$ to $9(39.2 \%)$ in the wild-type group; the mean age at CI was 4.0 years and 6.1 years, respectively. There were no significant differences in the sex or type of device implanted between the groups (the nucleus contour implant was used in all patients). The onset of bilateral pro- found SNHL was prelingual for all patients. All children had normal SMS test scores.

Ten patients who had an inner ear anomaly and/or developmental difficulties were excluded. The mean follow-up periods of the $G J B 2$ mutation and wild-type groups were 8.1 and 8.4 years, respectively. Twenty-eight patients showed normal radiological, neurological, psychological, and cognitive examination results. For the age-matched analysis, 14 patients were selected and divided into two groups: seven with a GJB2 mutation and seven with a wild-type gene (i.e., deafness of unknown origin). The control patients were carefully matched according to their age at the time of CI. Sixteen patients underwent $\mathrm{CI}$ before the age of 5 years. Among these cases, eight showed a GJB2 mutation and eight showed no mutation. The clinical features of both groups were similar (data not shown).

\section{Speech perception and language development}

In the analysis of the 14 age-matched patients, the GJB2 mutation and wild-type groups showed improvement after $\mathrm{CI}$ in terms of all speech reception test parameters. Scores at the 5-year follow-up in the mutation and wild-type groups were $94 \pm 7.9$ and $71.7 \pm 29.3$ in the open-set one-syllable test, $98.3 \pm 4$ and $95 \pm 12.3$ in the open-set two-syllable test, and $93 \pm 15.7$ and $89.6 \pm 15.1$ in the comprehension test, respectively. The mean MAIS score was $98.3 \pm 0.8$ in the mutation group and $98 \pm 0$ in the wild-type group at 5 years after CI. The CAP score was $7 \pm 0$ in the mutation group and $6.6 \pm 1.0$ in the wild-type group, and the SIR score was $3.9 \pm 0.6$ in the mutation group and $3.7 \pm 0.8$ in the wild-type group at 5 years after CI. There was no statistical difference in the speech perception test scores at the 5-year follow-up after CI between the mutation and wild-type groups. At the last follow-up (69 years) after CI, there was also no statistical difference in the speech perception test scores between the mutation and wild-type groups (Table 2, Fig. 1).

The scores of the speech reception tests in the patients who received CI before 5 years of age are shown in Table 3. These scores were significantly improved after CI. However, there

Table 1. Patient demographics and clinical characteristics

\begin{tabular}{lcc}
\hline \multicolumn{1}{c}{ Variables } & GJB2 mutation $(\mathrm{n}=15)$ & Wild type $(\mathrm{n}=23)$ \\
\hline Sex (male/female) & $12 / 3$ & $14 / 9$ \\
Age at operation (mean, years) & $1.0-13.4(4.0)$ & $1.1-14.6(6.1)$ \\
Type of device & Nucleus contour & Nucleus contour \\
Pure-tone average before implantation & 99 & 99 \\
Mutations identified in GJB2 genes & 235delC/235delC (4 patients) & None \\
Inner ear and congenital anomaly & E114G/E114G (11 patients) & 6 \\
Mean follow-up period (years) & 4 & 8.4 \\
\hline
\end{tabular}

GJB2: gap junction protein beta 2 
Table 2. Analysis of variance of the open-set, one- and two-syllable test, comprehension test, MAIS, SIR, and CAP scores at 5 and 6-9 years postoperatively in age-matched groups

\begin{tabular}{|c|c|c|c|c|c|}
\hline \multirow{2}{*}{ Speech perception test } & \multicolumn{2}{|c|}{ GJB2 (+) $(n=7)$} & \multicolumn{2}{|c|}{ Wild type $(n=7)$} & \multirow{2}{*}{$p$ value* } \\
\hline & $n$ & Mean \pm SD & $\mathrm{n}$ & Mean \pm SD & \\
\hline \multicolumn{6}{|l|}{ Open-set, one syllable } \\
\hline 5 years & 6 & $71.7 \pm 29.3$ & 7 & $94.3 \pm 7.9$ & 0.1669 \\
\hline Last FU (6-9 years) ${ }^{\dagger}$ & 7 & $78.6 \pm 14.6$ & 6 & $81.7 \pm 18.3$ & 0.4970 \\
\hline \multicolumn{6}{|l|}{ Open-set, two syllables } \\
\hline 5 years & 6 & $95.0 \pm 12.2$ & 6 & $98.3 \pm 4.1$ & 0.7665 \\
\hline Last FU (6-9 years) ${ }^{\dagger}$ & 7 & $88.6 \pm 26.1$ & 7 & $93.6 \pm 13.1$ & 0.9361 \\
\hline \multicolumn{6}{|l|}{ Comprehension test } \\
\hline 5 years & 5 & $89.6 \pm 15.1$ & 7 & $93.0 \pm 7.7$ & 0.8412 \\
\hline Last FU (6-9 years) ${ }^{\dagger}$ & 7 & $85.9 \pm 16.1$ & 6 & $89.2 \pm 8.9$ & 0.9426 \\
\hline \multicolumn{6}{|l|}{ MAIS } \\
\hline 5 years & 6 & $98.0 \pm 0$ & 7 & $98.3 \pm 0.8$ & 0.9764 \\
\hline Last FU (6-9 years $)^{\dagger}$ & 7 & $98.3 \pm 0.8$ & 6 & $98.3 \pm 0.8$ & 0.9093 \\
\hline \multicolumn{6}{|l|}{ SIR } \\
\hline 5 years & 6 & $3.7 \pm 0.8$ & 7 & $3.9 \pm 0.6$ & 0.9040 \\
\hline Last FU $(6-9 \text { years })^{\dagger}$ & 7 & $4.1 \pm 0.7$ & 6 & $4.5 \pm 0.5$ & 0.2539 \\
\hline \multicolumn{6}{|l|}{ CAP } \\
\hline 5 years & 6 & $6.6 \pm 1.0$ & 7 & $7.0 \pm 0$ & 0.6399 \\
\hline Last FU (6-9 years $)^{\dagger}$ & 6 & $6.8 \pm 0.6$ & 5 & $7.0 \pm 0$ & 0.3613 \\
\hline
\end{tabular}

* $p$ values were obtained using a linear mixed model at 5 years and the Mann-Whitney test at $6-9$ years, ${ }^{\dagger}$ the measures of the last follow-up from 6-9 years. n: number of patients, GJB2: gap junction protein beta 2, FU: follow-up, SD: standard deviation, MAIS: Meaningful Auditory Integration Scale, SIR: Speech Intelligibility Rating, CAP: Categories of Auditory Performance
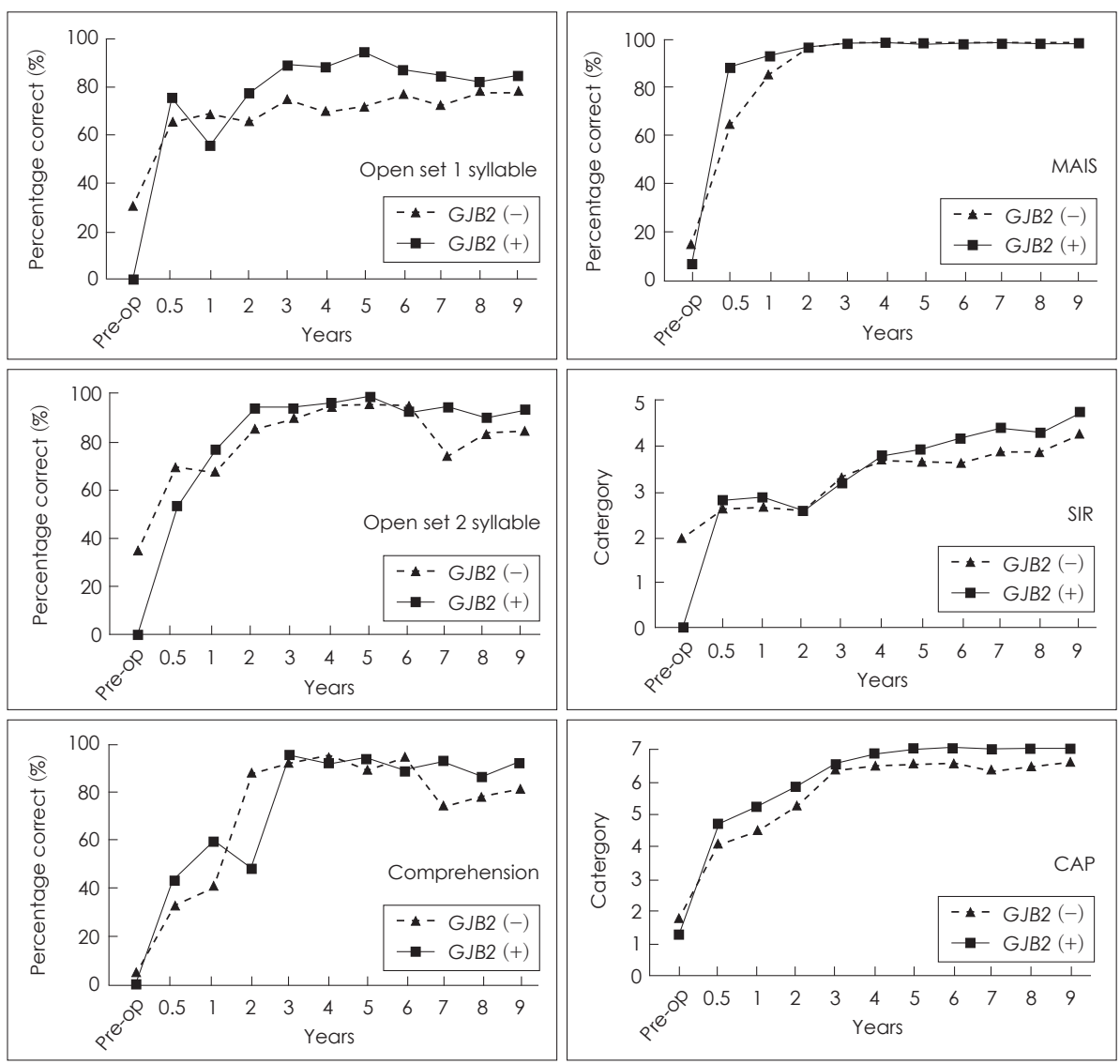

Fig. 1. Age matched. GJB2: gap junction protein beta 2, MAIS: Meaningful Auditory Integration Scale, SIR: Speech Intelligibility Rating, CAP: Categories of Auditory Performance.

98 J Audiol Otol 2017;21(2):95-102 
$\mathrm{Kim} \mathrm{SH}$, et al.

Table 3. Analysis of variance of the open-set, one- and two-syllable test, comprehension test, MAIS, SIR, and CAP scores at 5 and $6-9$ years postoperatively (age at surgery $<5$ years)

\begin{tabular}{|c|c|c|c|c|c|}
\hline \multirow{2}{*}{ Speech perception test } & \multicolumn{2}{|c|}{ GJB2 (+) $(n=8)$} & \multicolumn{2}{|c|}{ Wild type $(n=8)$} & \multirow{2}{*}{$p$ value* } \\
\hline & $\mathrm{n}$ & Mean \pm SD & $\mathrm{n}$ & Mean \pm SD & \\
\hline \multicolumn{6}{|l|}{ Open-set, one syllable } \\
\hline 5 years & 6 & $88.3 \pm 7.53$ & 7 & $97.1 \pm 4.9$ & 0.250 \\
\hline 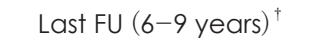 & 8 & $88.8 \pm 8.4$ & 8 & $93.8 \pm 5.2$ & 0.175 \\
\hline \multicolumn{6}{|l|}{ Open-set, two syllables } \\
\hline 5 years & 6 & $96.7 \pm 8.2$ & 7 & $98.6 \pm 3.8$ & 0.830 \\
\hline Last FU (6-9 years) ${ }^{\dagger}$ & 8 & $97.5 \pm 4.6$ & 8 & $98.8 \pm 3.5$ & 0.535 \\
\hline \multicolumn{6}{|l|}{ Comprehension } \\
\hline 5 years & 6 & $99.3 \pm 1.0$ & 7 & $96.3 \pm 4.2$ & 0.790 \\
\hline Last FU (6-9 years) ${ }^{\dagger}$ & 8 & $98.6 \pm 2.5$ & 8 & $97.4 \pm 3.2$ & 0.484 \\
\hline \multicolumn{6}{|l|}{ MAIS } \\
\hline 5 years & 6 & $98.0 \pm 0$ & 8 & $98.5 \pm 0.9$ & 0.927 \\
\hline 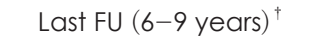 & 8 & $98.3 \pm 0.8$ & 8 & $98.5 \pm 0.9$ & 0.535 \\
\hline \multicolumn{6}{|l|}{ SIR } \\
\hline 5 years & 6 & $4.3 \pm 0.8$ & 8 & $4.5 \pm 0.5$ & 0.876 \\
\hline 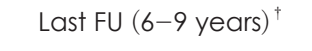 & 8 & $4.5 \pm 0.5$ & 8 & $4.9 \pm 0.2$ & 0.099 \\
\hline \multicolumn{6}{|l|}{ CAP } \\
\hline 5 years & 6 & $7.0 \pm 0$ & 7 & $7.0 \pm 0$ & 0.978 \\
\hline Last FU $(6-9 \text { years })^{\dagger}$ & 8 & $7.0 \pm 0$ & 7 & $7.0 \pm 0$ & $>0.999$ \\
\hline
\end{tabular}

* $p$ values were obtained using a linear mixed model at 5 years and the Mann-Whitney test at $6-9$ years, ${ }^{\dagger}$ the measures of the last follow-up from 6-9 years. n: number of patients, GJB2: gap junction protein beta 2, FU: follow-up, SD: standard deviation, MAIS: Meaningful Auditory Integration Scale, SIR: Speech Intelligibility Rating, CAP: Categories of Auditory Performance

Fig. 2. Op age <5 years. GJB2: gap junction protein beta 2, MAIS: Mean-ingful Auditory Integration Scale, SIR: Speech Intelligibility Rating, CAP: Categories of Auditory Performance.
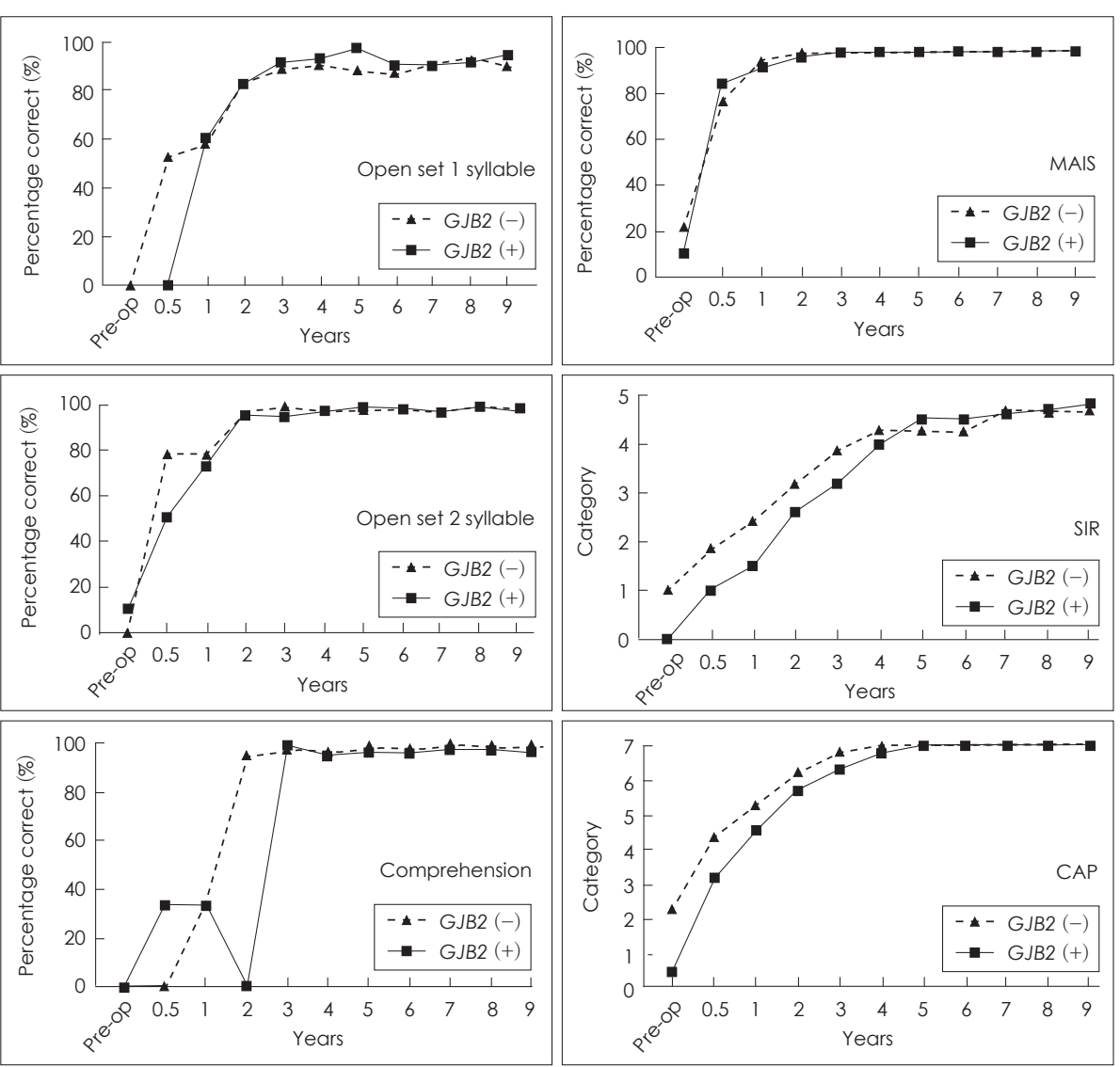
were no statistical differences between the GJB2 mutation and wild-type groups at the 5-year and last follow-ups (Table 3, Fig. 2).

The improvement pattern was also analyzed according to the follow-up time after CI. There was no statistically significant difference found in the pattern of longitudinal improvement in any speech reception parameters between the GJB2 mutation and wild-type groups in the age-matched analysis (Fig. 1) or in the analysis of patients who underwent CI before the age of 5 years (Fig. 2).
As a more practical approach, all speech perception results were transformed into RAU scores [20] to accommodate the ceiling effects demonstrated in the two groups (Table 4, 5). After a RAU transform on the data, there was a significant difference in the changing pattern over time in the mean MAIS score between the two groups in the age-matched analysis (Table 4). However, the group under 5 years of age showed no significant difference in the changing pattern over time and the overall speech perception scores between the GJB2 mutation and wild-type groups (Table 5). Table 6 shows the MAIS scores

Table 4. $p$ values of the variance of the open-set, one- and two-syllable test, comprehension test, MAIS, SIR, and CAP scores after a RAU transform on the data in age-matched groups

\begin{tabular}{lccc}
\hline \multirow{2}{*}{ Speech perception test } & p value for GJB2 mutation-by-time interaction* & \multicolumn{2}{c}{$p$ value for the overall effects ${ }^{\dagger}$} \\
\cline { 3 - 4 } & & \multicolumn{1}{c}{ Time } & GJB2 mutation \\
\hline RAU open-set, one syllable & 0.511 & 0.003 & 0.424 \\
RAU open-set, two syllables & 0.689 & $<0.001$ & 0.809 \\
RAU comprehension & 0.988 & $<0.001$ & 0.928 \\
RAU MAIS & 0.011 & & 0.984 \\
RAU SIR & 0.903 & $<0.001$ & 0.457 \\
RAU CAP & 0.318 & $<0.001$ & 0 \\
\hline
\end{tabular}

*GJB2 mutation-by-time interaction: the difference in the changing pattern over time between the GJB2 mutation and wild-type groups ( $p<0.05$ was considered to indicate statistical significance), ${ }^{\dagger}$ the $G J B 2$ mutation-by-time interaction effect was not statistically significant. GJB2: gap junction protein beta 2, MAIS: Meaningful Auditory Integration Scale, SIR: Speech Intelligibility Rating, CAP: Categories of Auditory Performance, RAU: rationalized arcsine unit

Table 5. $p$ values of the variance of the open-set, one- and two-syllable test, comprehension test, MAIS, SIR, and CAP scores after a RAU transform on the data (age at surgery $<5$ years)

\begin{tabular}{|c|c|c|c|}
\hline \multirow{2}{*}{ Speech perception test } & \multirow{2}{*}{ p value for GJB2 mutation-by-time interaction* } & \multicolumn{2}{|c|}{$p$ value for the overall effects ${ }^{\dagger}$} \\
\hline & & Time & GJB2 mutation \\
\hline RAU open-set, one syllable & 0.095 & $<0.001$ & 0.233 \\
\hline RAU open-set, two syllables & 0.815 & $<0.001$ & 0.488 \\
\hline RAU comprehension & 0.079 & 0.001 & 0.575 \\
\hline RAU MAIS & 0.155 & $<0.001$ & 0.617 \\
\hline RAU SIR & 0.531 & $<0.001$ & 0.266 \\
\hline RAU CAP & 0.862 & $<0.001$ & 0.066 \\
\hline
\end{tabular}

*GJB2 mutation-by-time interaction: the difference in the changing pattern over time between the GJB2 mutation and wild-type groups ( $p<0.05$ was considered to indicate statistical significance), ${ }^{\dagger}$ the $G J B 2$ mutation-by-time interaction effect was not statistically significant. GJB2: gap junction protein beta 2, MAIS: Meaningful Auditory Integration Scale, SIR: Speech Intelligibility Rating, CAP: Categories of Auditory Performance, RAU: rationalized arcsine unit

Table 6. $p$ values at each time point in the MAIS test between the GJB2 mutation and wild-type groups in the age-matched analysis

\begin{tabular}{|c|c|c|c|c|c|c|}
\hline \multirow{2}{*}{ Speech perception test } & \multirow{2}{*}{ Time* } & \multicolumn{2}{|c|}{ GJB2 $(+)(n=8)$} & \multicolumn{2}{|c|}{ Wild type $(n=8)$} & \multirow{2}{*}{$p$ value } \\
\hline & & $n$ & Mean $\pm S D$ & $n$ & Mean \pm SD & \\
\hline \multirow[t]{7}{*}{ RAU MAIS ${ }^{\dagger}$} & 1 & 5 & $51.6 \pm 14.8$ & 3 & $54.3 \pm 25.6$ & 0.709 \\
\hline & 6 & 6 & $92.6 \pm 6.5$ & 6 & $67.4 \pm 12.5$ & 0.023 \\
\hline & 12 & 6 & $99.5 \pm 5.3$ & 6 & $93.4 \pm 11.0$ & 0.046 \\
\hline & 24 & 6 & $106.0 \pm 2.3$ & 5 & $107.1 \pm 4.2$ & 0.742 \\
\hline & 36 & 7 & $109.8 \pm 1.4$ & 5 & $110.8 \pm 2.0$ & 0.978 \\
\hline & 48 & 7 & $111.2 \pm 1.8$ & 6 & $108.9 \pm 0.0$ & 0.790 \\
\hline & 60 & 7 & $109.8 \pm 1.4$ & 6 & $108.9 \pm 0.0$ & 0.894 \\
\hline
\end{tabular}

*months, ${ }^{\dagger} p$ values were obtained using a linear mixed model after a RAU transform on the data, ${ }^{\dagger}$ the GJB2 mutation-by-time interaction effect was statistically significant. MAIS: Meaningful Auditory Integration Scale, RAU: rationalized arcsine unit, GJB2: gap junction protein beta 2, SD: standard deviation, n: number of patients 
at each time point, and the mean MAIS score was significantly higher in the GJB2 mutation group $(92.6 \pm 6.5)$ than in the wild-type group (67.4 \pm 12.5$)$ at 6 months after $\mathrm{CI}(p=0.023)$.

\section{Discussion}

In the present study, we found no statistical differences in almost all the speech perception outcomes between the GJB2 mutation and wild-type groups of pediatric CI patients. In addition, these two groups showed similar longitudinal improvement.

Some studies have reported similar overall findings to those of our study [21-23]. Karamert, et al. [21], conducted a similar retrospective comparative study, and found no significant differences in the auditory performance of mutation-positive and -negative children after 1 year of follow-up as assessed by MAIS, the Meaningful Use of Speech Scale, and the LittIEARS Auditory Questionnaire. Yoshida, et al. [23], studied the results of long-term follow-up after CI in children with GJB2-related deafness in Japan. Their mean follow-up period after CI was 54.6 months in patients with no genetic abnormalities and 55.9 months in patients with GJB2-related deafness. They examined the speech performance outcomes before and after CI several times over a postoperative period ranging from 6 months to 4 years. The authors found no significant differences between the two groups in terms of hearing improvement, the Infant Toddler-MAIS score, development of articulation, and language perception and production.

Notably, however, there have also been reports of improved speech perception outcomes after CI and greater improvement in language expression tests in children with GJB2 mutations than in those with deafness of unknown etiology [2426]. Chora, et al. [24], studied a Portuguese CI cohort. They enrolled patients who had been implanted for longer than 5 years, and the mean duration of implant use was 7.72 years in the DFNB1 group and 8.90 years in the non-DFNB1 group. They reported $6 \%$ better global oral performance scores in patients with DFNB1-associated deafness than in those with deafness not associated with DFNB1 using the test designed by the Ear, Nose, and Throat team at Centro Hospitalar de Coimbra. Yan, et al. [26], reported significantly better outcomes as determined using the MAIS, CAP, and SIR tests over 2 years' follow-up after CI in patients with GJB2-related deafness than in cases with deafness of unknown etiology.

In our present study series, the follow-up period was 6-9 years. The data used to compare our two study groups were from the 5-year follow-up and the last follow-ups after CI. Because we had the longest mean follow-up period compared with other reports, we discovered that all speech parameters reached a maximum value at the 2- or 3-year follow-up (Fig. $1,2)$, and were maintained for $6-9$ years. This pattern of improvement implies that long-term performance is comparable in patients with or without a $G J B 2$ mutation, although speech performance may or may not differ during the first 2 or 3 years after CI. At the 3-year follow-up, the speech perception scores were already at the maximum values, and it was not possible to show any differences in the test results between the two study groups. From the results of this study, we could also conclude that the performances of both groups are sustained until long term follow up (up to 9 years).

The main limitation of our present analysis is that both groups exhibited ceiling effects. We performed a RAU transform on the data to move some subjects away from the ceiling in an attempt to reveal any differences between the two groups. Only the mean scores of the MAIS test were significantly better in the GJB2 mutation group than in the wild-type group at 6 months after CI.

The findings as presented here clearly show that both groups achieved high levels of performance in the open-set, one- and two-syllable tests, a comprehension test, MAIS, SIR, and CAP. However, we were unable to fully examine any differences in performance between the two groups with the chosen metrics for quantifying outcomes.

Other limitations were the small sample size and homogenous ethnicity of our subjects. Different gene mutations have varying effects that can depend on the ethnic background of the patients. In addition, the cause of deafness was unknown in our wild-type group. Further genetic studies are needed to elucidate the effect of gene mutations on deafness.

In conclusion, the long-term results of this study show no apparent performance differences between individuals with GJB2 mutations and a presumably heterogeneous group of individuals with hearing loss of unknown etiology. We were unable to find the difference between the two groups because all speech perception tests we performed already reached the highest point. The conflicting findings across the literature in this area could very well be because of the differences in the etiological composition of the "wild-type" sample. This confirms the need to perform further tests in a larger population of CI patients to ascertain the unknown etiologies of their deafness and also the influence of speech perception outcomes of CI.

\section{Conflicts of interest}

The authors have no financial conflicts of interest.

\section{REFERENCES}

1) Smith RJ, Bale JF Jr, White KR. Sensorineural hearing loss in chil- 
dren. Lancet 2005;365:879-90.

2) Lim HW, Kim EA, Chung JW. Audiological Follow-up Results after Newborn Hearing Screening Program. Clin Exp Otorhinolaryngol 2012;5:57-61.

3) Black J, Hickson L, Black B, Perry C. Prognostic indicators in paediatric cochlear implant surgery: a systematic literature review. Cochlear Implants Int 2011;12:67-93.

4) Kelsell DP, Dunlop J, Stevens HP, Lench NJ, Liang JN, Parry G, et al. Connexin 26 mutations in hereditary non-syndromic sensorineural deafness. Nature 1997;387:80-3.

5) Antoniadi T, Grønskov K, Sand A, Pampanos A, Brøndum-Nielsen $\mathrm{K}$, Petersen MB. Mutation analysis of the GJB2 (connexin 26) gene by DGGE in Greek patients with sensorineural deafness. Hum Mutat 2000;16:7-12.

6) Estivill X, Fortina P, Surrey S, Rabionet R, Melchionda S, D'Agruma $\mathrm{L}$, et al. Connexin-26 mutations in sporadic and inherited sensorineural deafness. Lancet 1998;351:394-8.

7) Gürtler N, Kim Y, Mhatre A, Müller R, Probst R, Lalwani AK. GJB2 mutations in the Swiss hearing impaired. Ear Hear 2003;24:440-7.

8) Lee KY, Choi SY, Bae JW, Kim S, Chung KW, Drayna D, et al. Molecular analysis of the GJB2, GJB6 and SLC26A4 genes in Korean deafness patients. Int J Pediatr Otorhinolaryngol 2008;72:1301-9.

9) Denoyelle F, Marlin S, Weil D, Moatti L, Chauvin P, Garabédian EN, et al. Clinical features of the prevalent form of childhood deafness, DFNB1, due to a connexin-26 gene defect: implications for genetic counselling. Lancet 1999;353:1298-303.

10) Murgia A, Orzan E, Polli R, Martella M, Vinanzi C, Leonardi E, et al. Cx26 deafness: mutation analysis and clinical variability. J Med Genet 1999;36:829-32.

11) Kikuchi T, Kimura RS, Paul DL, Adams JC. Gap junctions in the rat cochlea: immunohistochemical and ultrastructural analysis. Anat Embryol (Berl) 1995;191:101-18.

12) Lefebvre PP, Weber T, Rigo JM, Delree P, Leprince P, Moonen G. Potassium-induced release of an endogenous toxic activity for outer hair cells and auditory neurons in the cochlea: a new pathophysiological mechanism in Menière's disease? Hear Res 1990;47:83-93.

13) Kang WS, Lee JH, Lee HN, Lee KS. Cochlear implantations in young children with cochlear nerve deficiency diagnosed by MRI. Otolaryngol Head Neck Surg 2010;143:101-8.

14) Davcheva-Chakar M, Sukarova-Stefanovska E, Ivanovska V, Lazarevska V, Filipche I, Zafirovska B. Speech perception outcomes after cochlear implantation in children with GJB2/DFNB1 associated deafness. Balkan Med J 2014;31:60-3.

15) Popov TM, Stancheva I, Kachakova DL, Rangachev J, Konov D, Varbanova $\mathrm{S}$, et al. Auditory outcome after cochlear implantation in patients with congenital nonsyndromic hearing loss: influence of the GJB2 status. Otol Neurotol 2014;35:1361-5.

16) Kim LS, Lee MY, Huh MJ, Oh YJ. Open-set speech preception development in children with cochlear implants. Korean J Otorhinolaryngol-Head Neck Surg 2004;47:15-21.

17) Robbins AM, Renshaw JJ, Berry SW. Evaluating meaningful auditory integration in profoundly hearing-impaired children. Am J Otol 1991;12 Suppl:144-50.

18) Nikolopoulos TP, Archbold SM, Gregory S. Young deaf children with hearing aids or cochlear implants: early assessment package for monitoring progress. Int J Pediatr Otorhinolaryngol 2005;69:175-86.

19) Archbold S, Lutman ME, Nikolopoulos T. Categories of auditory performance: inter-user reliability. Br J Audiol 1998;32:7-12.

20) Studebaker GA. A "rationalized" arcsine transform. J Speech Hear Res 1985;28:455-62.

21) Karamert R, Bayazit YA, Altinyay S, Yılmaz A, Menevse A, Gokdogan $\mathrm{O}$, et al. Association of GJB2 gene mutation with cochlear implant performance in genetic non-syndromic hearing loss. Int J Pediatr Otorhinolaryngol 2011;75:1572-5.

22) Black J, Hickson L, Black B, Khan A. Paediatric cochlear implantation: adverse prognostic factors and trends from a review of 174 cases. Cochlear Implants Int 2014;15:62-77.

23) Yoshida H, Takahashi H, Kanda Y, Usami S. Long term speech perception after cochlear implant in pediatric patients with GJB2 mutations. Auris Nasus Larynx 2013;40:435-9.

24) Chora JR, Matos TD, Martins JH, Alves MC, Andrade SM, Silva LF, et al. DFNB1-associated deafness in Portuguese cochlear implant users: prevalence and impact on oral outcome. Int J Pediatr Otorhinolaryngol 2010;74:1135-9.

25) Varga L, Kabátová Z, Mašindová I, Nechojdomová D, Gašperíková D, Klimeš I, et al. Is deafness etiology important for prediction of functional outcomes in pediatric cochlear implantation? Acta Otolaryngol 2014;134:571-8.

26) Yan YJ, Li Y, Yang T, Huang Q, Wu H. The effect of GJB2 and SLC26A4 gene mutations on rehabilitative outcomes in pediatric cochlear implant patients. Eur Arch Otorhinolaryngol 2013;270:286570. 